\title{
OBJETIVOS E MÉTODOS DE PREÇO NO VAREJO: ESTUDO NA ZONA SUL DE SÃO PAULO
}

\author{
RETAIL PRICING STRATEGIES: STUDY IN THE SOUTH ZONE OF SÃO PAULO \\ OBJETIVOS Y MÉTODOS DE PRECIO EN EL MERCADO MINORISTA: ESTUDIO EN LA ZONA SUR DE SÃO PAULO
}

\section{RESUMO}

No varejo existe uma complexa tarefa na formulação das estratégias de precificação. Enquanto os objetivos de preço fornecem uma direção geral para ação, os métodos de precificação são procedimentos pelos quais as organizações atingem suas decisões de preços. O escopo deste trabalho é explorar os objetivos de preços do varejo juntamente com os métodos que adota para precificar, investigando se os objetivos estão associados aos métodos. A pesquisa mostra que as empresas da amostra estão fundamentalmente perseguindo objetivos quantitativos em vez de qualitativos, com uma forte ênfase na realização de vendas e lucros, seguido pelo desenvolvimento de mercado. Outro ponto relevante na discussão das estratégias de varejo: as empresas da amostra parecem dar menor importância a métodos de orientação ao cliente e empregam com mais frequência o método tradicional de custos.

PALAVRAS-CHAVE Marketing, marketing de varejo, estratégia varejista, objetivos de preço, métodos de preço.

Carlos Alberto Alves calves@uninove.br

Doutorando no Programa de Pós-Graduação em Administração da Universidade Nove de Julho - São Paulo - SP, Brasil

Luís Fernando Varotto luis.varotto@gvmail.com

Doutorando em Administração de Empresas pela Escola de Administração de Empresas de São Paulo, Fundação Getulio Vargas - São Paulo - SP, Brasil

Marcelo Neves Gonçalves marcelonevez@usp.br

Doutorando no Programa de Pós-Graduação em Administração da Universidade de São Paulo - SP, Brasil

\begin{abstract}
Retailers face a complex task in formulating prices strategies. While the goal of pricing is to lay down a general course of action, pricing methods are procedures which enable organizations to make their pricing decisions. The scope of this paper is to explore retail pricing goals along with the methods that are employed for pricing, and to investigate whether the methods employed succeed in achieving the objectives. It was found that the companies used in the sample are basically pursuing quantitative rather than qualitative objectives and that they lay emphasis on achieving sales and making profits by following market developments. Another significant factor was that, the firms in the sample seemed to attach less importance to customer-oriented methods and employ the traditional methods of costing, probably due to their simplicity.

Keywords Marketing, retail marketing, retail strategy, pricing objectives, pricing methods.

Resumen En el mercado minorista, la formulación de las estrategias de precificación es una compleja tarea. Mientras los objetivos de precio proporcionan una dirección general para la acción, los métodos de precificación son procedimientos por los cuales las organizaciones llegan a sus decisiones de precios. La intención de este trabajo es explorar los objetivos de precios del mercado minorista juntamente con los métodos que adopta para precificar, investigando si los objetivos están asociados a los métodos. El estudio muestra que las empresas de la muestra están persiguiendo fundamentalmente objetivos cuantitativos en vez de cualitativos, con fuerte énfasis en la realización de ventas y lucros, seguido por el desarrollo de mercado. Otro punto relevante en la discusión de las estrategias del mercado minorista: las empresas de la muestra parecen dar menor importancia a métodos de orientación al cliente y emplean con más frecuencia el método tradicional de costos.
\end{abstract}

Palabras clave Marketing, marketing minorista, estrategia minorista, objetivos de precio, métodos de precio. 


\section{INTRODUÇÃo}

No varejo, os gestores possuem uma tarefa delicada e complexa na formulação das suas estratégias de precificação, sobretudo considerando-se os múltiplos produtos disponíveis. Pesquisas anteriores sugerem que as estratégias de precificação dependem de fatores adicionais tais como: empresa, clientes, competição, regulamentação, entre outros (TELLIS, 1986).

A percepção de preço possui um impacto significante na satisfação do consumidor (KUKAR-KINNEY, RIDGWAY, MONROE, 2012; VARKI e COLGATE, 2001), o qual determina o desempenho nas vendas do varejo e a lealdade do cliente. Por outro lado, conforme os clientes revisitam uma loja, desenvolvem uma expectativa de preço ou um preço de referência, o qual se torna um parâmetro de comparação para os preços atuais (KUKAR-KINNEY, RIDGWAY, MONROE, 2012; POPESCU e WU, 2007). Preços acima da referência aparentam ser altos, preços abaixo da referência são percebidos como baixos. O efeito de preço baixo estimula a demanda de curto prazo e fornece incentivo para o varejista definir preços promocionais como mecanismo para incrementar os lucros de curto prazo. Preços promocionais diminuem as expectativas do consumidor e, portanto, sua boa vontade em adquirir produtos a preços mais altos no futuro. Para a empresa, isso significa que lucros maiores hoje vêm à custa da perda da demanda futura e, portanto, também lucros menores no futuro. Consequentemente, uma empresa que maximiza seus lucros deve considerar as implicações de longo prazo na determinação dos seus objetivos e métodos de precificação.

Autores como Diamantopoulos (1991), Indounas e Avlonitis (2009), Marn e Rosiello (1992), Simon (1992), Shipley e Jobber (2001) e Taher e Basha (2006) sinalizam a importância das decisões de preço para a lucratividade e sobrevivência de cada empresa no longo prazo. Marn e Rosiello (1992), Simon (1992) e Shipley e Jobber (2001) indicam que a precificação é o único elemento do composto de marketing que produz renda para a organização, enquanto os outros componentes estão relacionados às despesas. A precificação é também um dos seus elementos mais flexíveis, podendo ser alterado com rapidez (DIAMANTOPOULOS, 1991), afetando a competitividade, o volume de vendas, as margens e a lucratividade das empresas varejistas (INDOUNAS e AVLONITIS, 2009; TAHER e BASHA, 2006). Essa facilidade e flexibilida- de permitem que muitos varejistas atuem de maneira muito competitiva em sua resposta às ações da concorrência em termos de precificação. A consequência mais direta desse cenário é a adoção de métodos de preços pouco consistentes, de curto prazo, específicos e automáticos, sem planejamento prévio nem análise das consequências para o mercado e das expectativas do consumidor final (TOLEDO, PROENÇA, MELLO JR, 2006).

Considerando-se esses aspectos, o escopo deste trabalho é explorar os objetivos de preços que as empresas varejistas buscam juntamente com os métodos que adotam para precificar seus produtos e serviços, e, ainda, investigar se os objetivos de preço estão relacionados com os métodos de preço adotados.

\section{REFERENCIAL TEÓRICO}

A precificação no varejo enfatiza uma miríade de questões complexas que incluem a própria empresa varejista, o cliente, a concorrência, o contexto de mercado e a economia, entre outros fatores que precisam ser considerados pelos gestores do varejo. Segundo Cravens e Glover (1995), o primeiro passo para definir preços é determinar um objetivo de preço para a estratégia da empresa e, na sequência, determinar quais métodos de precificação serão empregados para atingir os objetivos de preços propostos.

\section{Objetivos de preço}

Os objetivos de preço fornecem direções para ação. Pesquisas empíricas indicam que as funções de preço das empresas de varejo são multifacetadas e que a viabilidade econômica das empresas repousa na combinação de diferentes objetivos de preço (DIAMANTOPOULOS, 1991).

As empresas devem avaliar diferentes objetivos e escolher suas próprias prioridades no contexto de problemas de precificação com que estejam se deparando, como declínio de vendas, preços mais altos ou baixos do que a concorrência, excessiva pressão sobre os intermediários para a geração de vendas, desbalanceamento de preços na linha de produtos, distorção quanto à percepção do consumidor em relação aos preços praticados pela empresa e mudanças frequentes de preços sem relação com a realidade do ambiente. 
Para Diamantopoulos (1991), os vários objetivos de preço podem ser classificados em dois grupos distintos: objetivos quantitativos e objetivos qualitativos, os quais estão resumidos no Quadro 1.

\section{Objetivos de preço quantitativos}

Os objetivos quantitativos podem ser facilmente medidos, pois incluem objetivos relacionados à lucratividade da empresa, vendas, fatia de mercado e cobertura dos custos. Revendo a literatura pertinente aos objetivos de preço quantitativos em empresas de serviços e varejo, foram identificados diversos constructos com as definições apresentadas no Quadro 2, mostrado a seguir.

\section{Objetivos de preço qualitativos}

Os objetivos de preço qualitativos são aqueles associados às metas menos quantificáveis, como relacionamento com os clientes, concorrentes, distribuidores, sobrevivência da empresa no longo prazo e atendimento de metas sociais. Revendo a literatura sobre objetivos de preço, o Quadro 3 apresenta um sumário dos principais objetivos de preço qualitativos em empresas de serviço e varejo.

As classificações de objetivos de preço apresentados acima, não obstante a complexidade das decisões de precificação, impõem a necessidade de perseguir mais de um objetivo por vez (OXENFELDT, 1983; DIAMANTOPOULOS, 1991; SMITH, 1995; JAIN, 2004). Além disso, nem todos os objetivos são compatíveis entre si, por exemplo, maximização das vendas pode levar a menores margens de lucro (KEIL, REIBSTEIN, WITTINK, 2001), enquanto uma ênfase excessiva nos lucros poderia ser contrastante com a obtenção de objetivos sociais.

\section{Métodos de precificação}

Enquanto os objetivos de preço fornecem uma direção geral para ação, os métodos de precificação são os passos ou procedimentos pelos quais as organizações atingem suas decisões de preço (MORRIS e CALANTONE, 1991, p. 322; OXENFELDT, 1983). Para Hansen e Solgaard (2004), torna-se essencial que a

\section{Quadro 1 - Objetivos de preço de empresas de varejo}

\begin{tabular}{|l|l|}
\hline \multicolumn{1}{|c|}{ Objetivos quantitativos } & \multicolumn{1}{c|}{ Objetivos qualitativos } \\
\hline Maximização do lucro & Liderança na qualidade dos serviços \\
\hline Maximização das vendas & Criação de uma imagem de prestigio \\
\hline Maximização da fatia de mercado & Para evitar uma guerra de preços \\
\hline Cobertura dos custos & Desenvolvimento de mercado \\
\hline Retorno sobre os investimentos feitos & Satisfação das necessidades dos consumidores \\
\hline Cobertura da capacidade existente & Similaridade de preços entre os concorrentes \\
\hline Diferenciação nos preços & Atração de novos clientes \\
\hline Satisfação das necessidades de distribuição & Realização de metas sociais \\
\hline Estabilidade dos preços no mercado & Desencorajar a entrada de novos competidores \\
\hline Estabilidade das vendas no mercado & Manutenção dos clientes existentes \\
\hline Incremento na fatia de mercado & Sobrevivência no longo prazo \\
\hline Retorno sobre os ativos da empresa & Determinação de um preço justo \\
\hline Realização e manutenção de liquidez & Realização de lucros em níveis satisfatórios \\
\hline & Realização de vendas em níveis satisfatórios \\
\hline
\end{tabular}

Fonte: DIAMANTOPOULOS, 1991 
formulação dos métodos de precificação dê suporte aos objetivos gerais da empresa e contenha uma flexibilidade embutida que torne possível uma adaptação imediata às mudanças ou novas condições que possam surgir no mercado.

Uma revisão da literatura dos métodos de precificação identificou 23 métodos empregados por empresas varejistas, que podem ser agrupados em três categorias básicas: (1) métodos baseados nos custos do ofertante; (2) métodos baseados nas expectativas do cliente; e (3) métodos baseados nas condições concorrenciais do mercado.

\section{Métodos baseados nos custos do ofertante}

Nos métodos baseados nos custos do ofertante, os preços são determinados com base nos custos para disponibilizar o produto ou serviço, os quais podem relacionar os custos de transporte, estocagem, promoção etc. Nesse tipo de estratégia, o preço não neces-

\section{Quadro 2 - Objetivos de preço quantitativos perseguidos pelas empresas de varejo}

\section{Objetivos de preço}

Incremento na fatia de mercado

Obtido pela alteração de preço para mais ou para menos, por ação promocional ou por estratégia de produtos

Procuram obter uma taxa específica de retorno sobre

Retorno sobre os investimentos

Maximização do lucro

Maximização de vendas

Maximização da fatia de mercado

Estabilidade dos preços no mercado

Cobertura dos custos

Estabilidade das vendas no mercado

Cobertura da capacidade existente

Retorno sobre os ativos da empresa

Realização e manutenção de liquidez da empresa

Diferenciação nos preços

Satisfação das necessidades de distribuição

\section{Caracteristicas principais} caracterizando-se como um objetivo de longo prazo desempenho da empresa no longo prazo

Maximização das receitas

Dominação do mercado por meio da determinação de de clientes dos concorrentes

Expectativa de retorno apropriado por uma atuação socialmente responsável perante a comunidade

Preços que permitam cobrir os custos incorridos na operação de varejo

Define preços iguais ou similares aos dos concorrentes diretos, estabilizando o preço dentro do mercado capacidade de atendimento existente

Procura obter retorno financeiro sobre os investimentos feitos em ativos na empresa

Procura vendas mais rápidas, garantindo um fluxo de liquidez constante clientes, serviços e locais

Leva em conta toda a cadeia de produção, aumentando a lucratividade do canal de distribuição
Principais autores

Kehagias, Skourtis e Vassilikopoulou (2009); Hornby e MacLeod (1996); Lanzillott, (1958); Levy e outros (2004).

Lanzillotti (1958); Shipley (1981).

Enfatiza o desempenho financeiro imediato mais do que 0 preços baixos a fim de tomar uma porcentagem significante

Determina os preços de serviços e produtos baseada na sua

Ajustar os preços básicos para acomodar diferentes tipos de
Hornby e MacLeod (1996).

Lanzillotti (1958).

Hornby e MacLeod (1996); Lanzillott, (1958); Kehagias, Skourtis e Vassilikopoulou (2009).

Lanzillotti (1958)

Shy (2008); Cravens e Glover (1995).

Lanzillotti (1958);

Shipley (1981); Moore (1995); Howard e Herbig (1996).

Lanzillotti (1958);

Shipley (1981); Howard e Herbig (1996).

Lanzillotti (1958).

Moore (1995); Howard e Herbig (1996).

Lanzillotti (1958).

Howard e Herbig (1996). 
sariamente negligencia as condições de mercado, mas o ponto de partida para a precificação está baseado nos custos, e as condições atuais de mercado não são consideradas até mais tarde.
Métodos baseados nas expectativas do cliente

No método de preço baseado nas expectativas do cliente, o propósito primário da precificação é naturalmente gerar ganhos para a empresa. A empresa deve

\section{Quadro 3 - Objetivos de preço qualitativos perseguidos pelas empresas de varejo}

Objetivos de preço
$\begin{aligned} & \text { Similaridade de preços com a } \\ & \text { concorrência }\end{aligned}$
Liderança na qualidade dos
serviços
Criação de uma imagem de
prestigio
Satisfação das necessidades
dos consumidores
Sobrevivência da empresa no
longo prazo

Evitar uma guerra de preços

Desenvolvimento de mercado

Atração de novos clientes

Manutenção dos clientes existentes

Realização de metas sociais

Desencorajar a entrada de novos concorrentes

Realização de fatia de mercado em níveis satisfatórios

Preço justo

Realização de lucros em níveis satisfatórios

Realização de vendas em níveis satisfatórios

\section{Caracteristicas principais}

Cobra preços iguais ou similares aos dos concorrentes diretos

Justificado pela alta qualidade dos serviços prestados e os altos custos no desenvolvimento do serviço

Preços altos combinados com alta qualidade

Combinação de serviços e preços que atenda as necessidades únicas de cada grupo de clientes

Preparar seus recursos para as contingências por meio de objetivos de preços apropriados

Procuram evitar que a concorrência reaja de modo muito forte aos preços

Define o preço com o objetivo de desenvolver o mercado para determinado produto. Normalmente um preço um pouco mais baixo do que 0 ideal

Preços altos ou baixos em conformidade com o perfil do cliente que se quer atrair

Objetivo de precificação é considerado a satisfação e lealdade dos clientes

Objetivos de preço de forma a atender metas que a sociedade implicitamente institui

Manter os preços estáveis conjuntamente com os atuais concorrentes de forma a evitar que novos concorrentes surjam no setor

Procura obter uma fatia de mercado, por meio do ajuste de preços e que seja suficiente para a manutenção das suas operações

Tentativa da empresa em conseguir uma boa imagem perante o mercado, a comunidade e os consumidores. Evitar abusos com relação a aumento de preços em situações socialmente adversas: como crises de abastecimento; desastres naturais etc.

Obter lucros que satisfaçam as suas necessidades econômicas de médio e longo prazo

Empresa procura por vendas que satisfaçam as suas necessidades econômicas de médio e longo prazos

\section{Principais autores}

Lanzillotti (1958); Shipley (1981); Howard e Herbig (1996).

Cravens e Glover (1995); Howard e Herbig (1996); Hornby e Macleod (1996); Hoffman e Bateson (2010).

Howard e Herbig (1996).

Moore (1995).

Cravens e Glover (1995).

Moore (1995); Howard e Herbig (1996).

Lanzillotti (1958); Moore (1995); Howard e Herbig (1996).

Lanzillotti (1958); Matzler e Hinterhuber (1998).

Lanzillotti (1958); Matzler e Hinterhuber (1998).

Cravens e Glover (1995); Sirgy e Dong-Jin (1996).

Lanzillotti (1958); Kehagias, Skourtis e Vassilikopoulou (2009).

Hornby e MacLeod (1996); Kehagias, Skourtis e Vassilikopoulou (2009); Lanzillotti (1958).

Shipley (1981); Howard e Herbig (1996); Lan, Kukar-Kinney e Monroe (2010); Theotokis, Pramatari e Tsiros (2012).

Hornby e MacLeod (1996).

Hornby e MacLeod (1996). 


\section{Quadro 4 - Métodos baseados nos custos do ofertante}

\section{Métodos de preço}

Preço para pacote misto

Preço por imagem

Preço por captura ou preço por acessórios

Preço alto ou premium princing

Preço de desnatamento ou skimming price

Preço por pacote

Preço por eficiência

\section{Caracteristicas principais}

Tipo especial de pacote no qual dois ou mais produtos que podem ser comprados separadamente são oferecidos conjuntamente com um preço reduzido

Oferece-se um produto que possui a mesma imagem de outro produto, capturando ou transferindo seus atributos

Oferece-se o produto central com preços mais baixos quando os itens ou acessórios, suprimentos e serviços podem ser precificados a um valor mais alto

Oferece-se uma versão do produto com atributos superiores (qualidade, nome, entre outros) e que 0 consumidor aceita pagar 0 prêmio pela compra desses atributos

Produto novo no mercado sem nenhum produto similar ou concorrente, é possível definir um preço além dos custos mantendo uma margem mais alta de lucro, conforme a movimentação da concorrência e a introdução de produtos ou serviços similares diminuem-se gradualmente os preços, mantendo a concorrência sob controle

Apresenta-se o produto como parte de um pacote com diversos outros produtos que são mais baratos do que a soma de preços individuais

Conseguir eficiência na operação e repassar esses ganhos em forma de preços mais baixos para o consumidor

\section{Principais autores}

Guiltinam (1987); Manning e Sprott (2007); Munger e Grewal (2001).

Rao e Monroe (1996); Hawkins (1954); Tellis (1986); Zielke (2006, 2010).

Noble e Gruca (1999).

Hawkins (1954); Rao e Monroe (1996); Yeoman e McMahon-Beattie (2006).

Calantone e Di Benedetto (2007); Hawkins (1954); Tellis (1986).

Guiltinam (1987); Munger e Grewal (2001).

Berry e Yadav (1996).

\section{Quadro 5 - Métodos baseados nas expectativas do cliente}

\section{Métodos de preço}

Preço único ou preço de lista

Preço negociado

Preço por relacionamento

Preço chamariz

Preço por diferenciação

Preço baixo todo dia

Preço psicológico

\section{Caracteristicas principais}

Define-se o preço para o produto e procura manter esses preços ao longo do tempo

Cliente negocia o preço de venda com o varejista

Oferecem-se preços especiais para criar ou manter um relacionamento com o cliente

Em alguns produtos especiais, preços abaixo do custo ou preços relativamente baixos para atrair 0 cliente ao local de venda

Procura-se por formas de criar diferenciais no preço com relação aos mesmos produtos oferecidos pela concorrência

Preços onde o varejo tenta convencer o consumidor de que os preços entre todos os produtos do portfólio são consistentemente baixos

Define-se um preço pouco abaixo de um valor inteiro $(\mathrm{R} \$ 0,99)$

\section{Principais autores}

Hawkins (1954).

Evans e Beltramini (1987).

Berry e Yadav (1996).

DeGraba (2006); Lal (1991); Lal e Matutes (1994); Hess e Gerstner (1987).

Hawkins (1954); Lal (1991); Tellis (1986).

Marinescu, Mihai e Sorin, (2010).

Anderson e Simester (2003); Bray e Harris (2006); Hawkins (1954); Macé (2012); Thomas e Morwitz (2005). 
intuitivamente tentar determinar um preço que maximize os ganhos sobre um curto ou longo espaço de tempo de acordo com seus objetivos. A precificação baseada nas expectativas do cliente está em acordo com a concepção do mercado o qual exprime que o ponto de partida para a precificação está baseado nos desejos e necessidades dos consumidores.

\section{Métodos decorrentes das condições concorrenciais do mercado}

No método de preço baseado nas condições concorrenciais do mercado, o propósito é gerar demanda para a empresa, considerando a função de demanda e outros fatores como competição, questões legais etc.

\section{Quadro 6 - Métodos decorrentes das condições concorrenciais do mercado}

Métodos de preço

Preço por quantidade

Preço por localidade ou geografia

Descontos comerciais

Preço por pagamento em dinheiro

Preço de penetração

Preço de escoamento

Preço de acordo com as necessidades dos clientes
Características principais

Principais autores

Ganha-se economia de escala com uma venda superior, oferecendo em troca descontos pelas quantidades adquiridas

Preço do produto é diferente para diferentes mercados geográficos

Periodicamente oferecem-se descontos de preço para este produto

Oferece-se um desconto para os clientes que optam pelo pagamento em dinheiro

Define-se um preço inicial baixo para o produto para acelerar a sua adoção pelos consumidores; se ganha mercado dos seus concorrentes, ao fim da promoção, os preços são redefinidos nos patamares normais do mercado.

Procura-se por todas as formas acelerar a venda dos produtos, seja por meio de descontos promocionais, seja por meio de atividades que facilitam 0 acesso do consumidor aos produtos

Preço é determinado para satisfazer as necessidades dos consumidores
Chen Moorthy e Zhang (2005);

Hawkins (1954); Shah e Dixit (2005).

Guthrie (1950); Seidler (1937).

Chen, Moorthy e Zhang (2005); Shah e Dixit (2005).

Chen Moorthy e Zhang (2005); Langeard (2000); Shah e Dixit (2005).

Calantone e Di Benedetto (2007); Tellis (1986).

Chen, Moorthy e Zhang (2005); Tellis (1986).

Bonnici (1991); Ratza (1993).

\section{Tabela 1 - Divisão das equipes de pesquisa por bairros e resultado dos questionários recebidos}

\begin{tabular}{|l|l|c|c|}
\hline \multicolumn{1}{|c|}{ Equipes } & \multicolumn{1}{|c|}{ Bairros } & Questionários entregues/retornados & Taxa percentual de retorno \\
\hline Equipe_01 & Santo Amaro, Socorro & $592 / 122$ & $20,61 \%$ \\
\hline Equipe_02 & Campo Belo e Campo Limpo & $261 / 79$ & $30,27 \%$ \\
\hline Equipe_03 & Moema, Morumbi, Capão Redondo & $426 / 39$ & $8,45 \%$ \\
\hline Equipe_04 & Socorro, Vila Sônia & $260 / 61$ & $23,46 \%$ \\
\hline Equipe_05 & Jabaquara, Paraisópolis, Cursino & $521 / 79$ & $15,16 \%$ \\
\hline Equipe_06 & Interlagos, Parelheiros & $308 / 57$ & $18,51 \%$ \\
\hline Total geral & & $2.368 / 437$ & $18,45 \%$
\end{tabular}


Os três grupos de métodos de precificação - baseado nos custos do ofertante, baseado nas expectativas do cliente e baseado nas condições concorrenciais do mercado - não devem ser considerados separadamente uns dos outros. No método baseado nos custos do ofertante, naturalmente considerações sobre condições concorrenciais do mercado e expectativas do cliente são incluídos, bem como precificação baseada nas expectativas do cliente também considera questões sobre condições concorrenciais do mercado e os custos do ofertante. A divisão em três grupos de métodos refere-se apenas ao ponto de partida para os métodos de precificação da empresa.

\section{PERSPECTIVA METOdOLÓGICA}

Para responder aos objetivos deste trabalho, optou-se por realizar uma pesquisa de cunho quantitativo-descritivo por meio de levantamento do método tipo survey.

\section{Amostras e coleta de dados}

A seleção da amostra é não probabilística e por conveniência (KRZANOWSKI, 2007); a escolha por amostra não probabilística deu-se devido ao grande número de estabelecimentos varejistas espalhados na cidade de São Paulo e à dificuldade em conseguir aleatoriamente um contato para a realização da pesquisa. Outros fatores que impactaram na decisão pela amostra não probabilística foram: limitação orçamentária e limitação de tempo. A coleta de dados ocorreu entre os meses de abril e junho de 2011. O levantamento foi realizado por seis equipes de pesquisa, cada equipe possuía entre 4 e 8 indivíduos, fazendo um total de 35 pessoas. A área pesquisada constituía-se de cen-

\section{Tabela 2 - Porte das empresas varejistas participantes da pesquisa}

\begin{tabular}{|l|c|c|}
\hline & N & $\%$ \\
\hline Grande & 99 & 32,9 \\
\hline Média & 62 & 20,6 \\
\hline Pequena & 58 & 19,3 \\
\hline Micro & 81 & 27,2 \\
\hline Total de empresas respondentes & 300 & 100,0 \\
\hline
\end{tabular}

tros comerciais de bairro, classificação apresentada por Entenberg (1960), todos da zona sul da cidade de São Paulo. Foram visitados 2.368 estabelecimentos para entrega do instrumento de pesquisa. Dos estabelecimentos visitados, 437 aceitaram responder ao instrumento, uma taxa de 18,45\%. Dos questionários retornados, apenas 300 foram empregados na análise, 137 foram descartados por possuírem algum tipo de erro que inviabilizava seu uso, como falta de resposta em alguns dos itens ou duplo preenchimento, entre outros problemas percebidos. Um resumo descritivo é apresentado na Tabela 1 , e os dados obtidos foram tabulados no software SPSS v.19.

\section{Instrumento de pesquisa}

Os dados foram obtidos por meio de um questionário de quatro páginas. A escala escolhida para uso neste estudo foi a desenvolvida por Indounas e Avlonitis (2009). Seguindo as sugestões de Herzog e Bachman (1981), esforços foram feitos para evitar questões ambíguas, prestando-se muita atenção na sequência de questões e assegurando um formato e estilo profissionais.

\begin{tabular}{|l|c|c|}
\hline \multicolumn{3}{|c|}{ Tabela 3 - Composição dos funcionários } \\
\hline & N & $\%$ \\
\hline Acima de 100 funcionários & 97 & 32,2 \\
\hline De 50 a 99 funcionários & 45 & 15,0 \\
\hline De 10 a 49 funcionários & 59 & 19,6 \\
\hline Até 9 funcionários & 99 & 33,2 \\
\hline Total de empresas respondentes & 300 & 100,0 \\
\hline
\end{tabular}

\section{Tabela 4 - Composição hierárquica dos respondentes}

\begin{tabular}{|l|c|c|}
\hline & N & $\%$ \\
\hline Analista & 14 & 4,7 \\
\hline Coordenador & 45 & 15,0 \\
\hline Gerente & 183 & 60,8 \\
\hline Diretor & 25 & 8,3 \\
\hline Presidente & 33 & 11,3 \\
\hline Total de empresas respondentes & 300 & 100,0 \\
\hline
\end{tabular}




\section{Tabela 5 - Descrição geral do tipo de varejo}

\begin{tabular}{|l|c|c|}
\hline & N & $\%$ \\
\hline Outros tipos de varejo & 40 & 13,3 \\
\hline Varejo de armarinhos & 6 & 2,3 \\
\hline Varejo cama, mesa e banho & 4 & 1,3 \\
\hline Varejo de informática e telecomunicações & 8 & 2,7 \\
\hline Varejo de mat. construção e manutenção & 7 & 2,3 \\
\hline Varejo de saúde & 49 & 16,3 \\
\hline Varejo de vestuário & 142 & 47,2 \\
\hline Varejo de alimento & 44 & 14,6 \\
\hline Total de empresas respondentes & 300 & 100,0 \\
\hline
\end{tabular}

Para fazer equivalências idiomática, semântica, cultural e conceitual, os conceitos foram repassados simultaneamente, na íntegra e em língua original (inglês), a dois tradutores independentes sem o conhecimento prévio sobre a pesquisa. Obtiveram-se duas traduções distintas, denominadas tradução 1 e 2. Essas traduções foram examinadas por um comitê constituído por pesquisadores, o qual leu e realizou adaptações idiomáticas e semânticas próprias da linguagem técnica e retrotraduziu para o inglês, comparando-as às originais. Não houve diferenças significativas, e continuou-se a validação. A validação de face do instrumento foi realizada pelo mesmo comitê, e com uma comparação entre as traduções

\section{Tabela 6 - Análise fatorial exploratória e componentes resultantes}

\begin{tabular}{|c|c|c|c|c|c|}
\hline \multirow[b]{2}{*}{ Itens } & \multirow[b]{2}{*}{ Média } & \multirow[b]{2}{*}{$\begin{array}{l}\text { Desvio } \\
\text { Padrão }\end{array}$} & \multicolumn{3}{|c|}{ Componentes } \\
\hline & & & $\begin{array}{l}\text { Objetivos de de- } \\
\text { senvolvimento } \\
\text { de mercado }\end{array}$ & $\begin{array}{l}\text { Objetivos de } \\
\text { realização de } \\
\text { lucros e vendas }\end{array}$ & $\begin{array}{l}\text { Objetivos } \\
\text { de com- } \\
\text { petiçãa }\end{array}$ \\
\hline Var_a21 - Atração de novos clientes para a empresa & 4,47 & 0,815 & 0,739 & & \\
\hline Var_a18 - Desenvolvimento de mercado para a empresa & 4,12 & 0,871 & 0,732 & & \\
\hline Var_a12 - Incremento na fatia de mercado & 4,06 & 0,873 & 0,668 & & \\
\hline $\begin{array}{l}\text { Var_a11 - Realização de fatia de mercado em níveis } \\
\text { satisfatórios para a empresa }\end{array}$ & 4,11 & 0,843 & 0,663 & & \\
\hline Var_a3 - Maximização da fatia de mercado & 4,10 & 0,857 & 0,582 & & \\
\hline Var_a6 - Cobertura da capacidade existente & 4,01 & 0,838 & 0,566 & & \\
\hline Var_a19 - Satisfação das necessidades dos consumidores & 4,44 & 0,830 & 0,475 & & \\
\hline Var_a2 - Maximização das vendas & 4,51 & 0,677 & 0,403 & & \\
\hline $\begin{array}{l}\text { Var_a27 - Realização de lucros em níveis satisfatórios } \\
\text { para a empresa }\end{array}$ & 4,57 & 0,664 & & 0,881 & \\
\hline $\begin{array}{l}\text { Var_a28 - Realização de vendas em níveis satisfatórios } \\
\text { para a empresa }\end{array}$ & 4,62 & 0,631 & & 0,836 & \\
\hline $\begin{array}{l}\text { Var_a20 - Similaridade de preços entre os concorrentes e } \\
\text { os preços }\end{array}$ & 3,81 & 1,082 & & & 0,790 \\
\hline $\begin{array}{l}\text { Var_a17 - Para evitar uma guerra de preços entre a } \\
\text { empresa e os concorrentes }\end{array}$ & 3,60 & 1,125 & & & 0,776 \\
\hline Var_a23 - Desencorajar a entrada de novos competidores & 3,23 & 1,328 & & & 0,569 \\
\hline Autovalores & & & 4,642 & 1,479 & 1,121 \\
\hline Alfa de Cronbach & & & 0,821 & 0,821 & 0,596 \\
\hline Total da Variância Explicada (\%) & & & 33,159 & 10,565 & 8,007 \\
\hline Total da Variância Acumulada (\%) & & & 33,159 & 43,724 & 51,732 \\
\hline
\end{tabular}


1 e 2 e a versão original obteve-se a versão final, resultado da fusão de 1 e 2 .

Além disso, antes de empregar o instrumento para a coleta de dados, um pré-teste foi realizado, baseado em entrevistas pessoais entre dois acadêmicos e 10 gestores da área de varejo, a fim de validar o instrumento. As inconsistências e ambiguidades do instrumento de pesquisa foram corrigidas e eliminadas antes do trabalho de campo (MALHOTRA, 2001).

\section{Medidas}

Os objetivos de preços foram medidos por meio de 28 questões, utilizando escala de Likert de cinco pontos, indo de Discordo Totalmente $=1$ a Concordo Totalmente $=5$, de acordo com a importância que o objetivo de preço possuía para a organização.

Os métodos de precificação foram medidos por meio de 12 questões, utilizando uma escala dicotômica: Emprega-se $=1$ e Não se emprega $=0$. Os respondentes poderiam optar por mais de um método de precificação.

\section{RESULTADOS E ANÁLISES}

Das 300 empresas que participaram desse projeto de pesquisa, nota-se, conforme mostrado na Tabela 2, que a grande maioria era constituída de grandes e microempresas (33\% e 27\%) respectivamente, seguida pelas médias empresas $(20,6 \%)$ e pequenas empresas (19,3\%). A divisão entre micro, pequenas, médias e grandes empresas obedeceu à classificação empregada pelo Serviço de Apoio às Micro e Pequenas Empresas de São Paulo (Sebrae-SP) (BEDÊ, 2006).

Os valores sobre o porte das empresas discutidos anteriormente estão de acordo com os valores sobre o número de funcionários, mostrado na Tabela 3 , que é outra medida utilizada para descrever o porte da empresa. Empresas com mais de 100 funcionários representavam 32,2\% (grandes empresas) da amostra e empresas com até 9 funcionários representavam 33\% (micro empresas), porém, no estrato de médias e pequenas empresas, essa relação não se confirmou: empresas que iam de 50 a 99 funcionários representavam $15 \%$ da amostra e empresas que iam de 10 a 49 funcionários representavam 19,6 $\%$ da amostra.

Como mostrado na Tabela 4, a maioria dos respondentes classificou-se como: gerentes, $61 \%$, coor- denadores, $15 \%$ e presidentes ou proprietários, 11\% dos entrevistados.

Com relação ao tipo de varejo, pode-se notar uma predominância do varejo de vestuário, com $47,2 \%$ das amostras, seguido pelo varejo de saúde, com 16,3\%, e varejo alimentício, com 14,6\%, de acordo com a Tabela 5 , O restante ficou divido entre os outros tipos de varejo: armarinhos; cama, mesa e banho; informática e telecomunicações; e materiais para construção e manutenção.

\section{Objetivos de preço}

Na Tabela 6, são mostradas as médias para cada um dos objetivos de preço. Pode-se notar que os três ob-

\begin{tabular}{|l|c|c|}
\hline \multicolumn{2}{|c|}{$\begin{array}{l}\text { Tabela 7 - Métodos de preço adotados } \\
\text { por empresas de varejo }\end{array}$} \\
\hline Item & N & $\%$ \\
\hline Pesconto por quantidade & 154 & $51 \%$ \\
\hline Desconto negociado & 152 & $50 \%$ \\
\hline Descontos comerciais & 150 & $50 \%$ \\
\hline Preço chamariz & 137 & $46 \%$ \\
\hline Preço psicológico & 137 & $46 \%$ \\
\hline Preço para escoamento (venda rápida) & 124 & $41 \%$ \\
\hline Preço por diferenciação & 111 & $37 \%$ \\
\hline Preço por localidade ou geografia & 107 & $36 \%$ \\
\hline Preço único & 106 & $35 \%$ \\
\hline Preço para entrar no mercado & 105 & $35 \%$ \\
\hline Preço por relacionamento & 104 & $35 \%$ \\
\hline Preço por imagem & 81 & $27 \%$ \\
\hline Preço baixo todo dia & 80 & $27 \%$ \\
\hline Preço por acessórios & 71 & $24 \%$ \\
\hline Preço de penetração & 67 & $22 \%$ \\
\hline Preço por serviço agregado & 66 & $22 \%$ \\
\hline Preço para pacote misto & 64 & $21 \%$ \\
\hline Preço por captura & 55 & $18 \%$ \\
\hline Preço por eficiência & 55 & $18 \%$ \\
\hline Desnatamento de preço & 48 & $16 \%$ \\
\hline & 40 & $13 \%$ \\
\hline
\end{tabular}

Nota: a soma total das porcentagens é maior do que 100, já que uma empresa pode utilizar mais de um método de preço. 
jetivos de preço mais importantes são aqueles relacionados às vendas e aos lucros, mais especificamente: realização de vendas em níveis satisfatórios para a empresa $(4,62)$; realização de lucros em níveis satisfatórios para a empresa (4,57); e maximização das vendas $(4,51)$. Esses achados são consistentes com os objetivos quantitativos de preço, apresentados previamente no corpo do referencial teórico.

Uma Análise Fatorial Exploratória (AFE) com análise de componente principal e rotação Varimax, Autovalor igual a 1 e carga fatorial maior do que 0,4 foi realizada e está apresentada na Tabela 6 , mostrada a seguir. Os resultados obtidos após aplicação da AFE indicam três componentes ou fatores finais, definidos como: objetivos de desenvolvimento de mercado; objetivos de realização de vendas e lucros; e objetivos de competição.

Alguns itens foram eliminados com base em critérios combinados ou utilizados isoladamente. Se um item possuísse carga fatorial inferior a 0,4 , poderia ser eliminado, assim como se possuísse carga fatorial superior a 0,4 em mais de um fator. Um item também poderia ser eliminado se ele se relacionasse diretamente a um fator mal definido, com poucos itens ou com um Alfa de Cronbach muito baixo (menor do que 0,5). Após a eliminação de qualquer item, a análise fatorial era novamente calculada para verificação dos resultados e adequação das validades.

Os fatores representam diferentes objetivos de preço e explicam 51,73\% do total da variância, enquanto a confiabilidade para cada fator medido pelo Alfa de Cronbach variou de 0,596 a 0,821, o qual pode ser considerado satisfatório (NUNNALLY, 1978). Além do que, esses valores estão consistentes com os esquemas classificatórios desenvolvidos por outros autores (HAWKINS, 1954).

\section{Métodos de preço}

A distribuição para cada método de preço é mostrado na Tabela 7, e pode-se notar que os métodos mais populares são: desconto por quantidade; preço negociado; e desconto por pagamento em dinheiro. $\mathrm{O}$ emprego desses métodos é condizente com o setor varejista e também pode ser atribuído ao fato de serem simples de se implantar.

\section{Associação entre objetivos de preço e métodos de preço}

Para descobrir se existe uma associação entre objetivos e os métodos de preço, foi realizada uma análise de regressão logística. Essa análise é uma regressão múltipla, porém com a variável de saída sendo dicotômica categórica e as variáveis preditoras sendo contínuas ou categóricas (FIELD, 2009; HAIR e outros, 2009).

A análise por regressão logística foi empregada para examinar o impacto dos objetivos de preço definidos pela empresa nos métodos adotados, dado que os métodos de precificação, nesse estudo, são variáveis categóricas, enquanto os objetivos de preço são variáveis contínuas. Mais especificamente, os métodos de precificação são considerados variáveis dependentes, enquanto os objetivos de preço são variáveis independentes. Em outras palavras, examina-se a adoção de

\section{Tabela 8 - Preço para escoamento ou venda rápida e objetivos de preços}

\begin{tabular}{|l|c|c|c|c|}
\hline & Coeficiente $\beta$ (EP) & \multicolumn{3}{|c|}{ Intervalo de confiança de 95\% para Exp( $\beta)$} \\
\hline Objetivos de desenvolvimento de mercado & $0,057(0,56)$ & 0,872 & 1,058 & 1,285 \\
\hline Objetivos de realização de lucros e vendas & $0,128(0,2)$ & 0,932 & 1,137 & 1,387 \\
\hline Objetivos de competição & $0,034(0,71)$ & 0,863 & 1,035 & 1,241 \\
\hline Porcentagem total das predições corretas & $49,7 \%$ & & \\
\hline Log Máxima verossimilhança (max) & 414,04 & & \\
\hline $\mathrm{R}^{2}$ de Nagelkerke & 0,008 & & \\
\hline$\chi^{2}$ melhoramento & $26,491, \mathrm{gl:} 8$ & \\
\hline
\end{tabular}

Nota: $p<0,05, p<0,01$, EP - Erro Padrão; gl - graus de liberdade 
um método em particular como uma função dos objetivos de preço. Para se evitar a possibilidade da multicolinearidade, os 13 fatores multidimensionais dos objetivos de preço, os quais são derivados da análise fatorial, são utilizados como variáveis independentes.

Após realizar 11 regressões logísticas, uma para cada método de precificação, cinco foram consideradas estatisticamente significantes.

\section{Preço para escoamento ou venda rápida}

A análise pertinente ao preço para escoamento rápido ou venda rápida (Tabela 8) revelou que apenas um objetivo de preço está associado com esse método de precificação.

O objetivo de realização de lucros e vendas possui uma associação positiva $(0,128)$ com o método de preço para escoamento rápido. Isso está de acordo com a teoria desenvolvida por Tellis (1986), já que esse tipo de método é mais voltado a resultados quantitativos na realização de lucros e vendas. No método de esco- amento, como o varejista procura formas de realizar vendas rápidas, a associação com os lucros é natural. A respectiva matriz de correlação entre os objetivos de preço é mostrada na Tabela 9, a seguir.

\section{Preço por diferenciação}

Com referência ao método de preço por diferenciação (Tabela 10), a possibilidade da sua adoção aumenta quando os objetivos perseguidos pela empresa são relacionados à competição $(0,620)$ e à realização de lucros e vendas $(0,119)$.

A diferenciação ocorre como meio de obter maiores lucros e vendas quando a organização se posiciona perante o mercado ou perante a concorrência. Esse método está de acordo com o que foi definido por pesquisadores como Hawkins (1954), Lal (1991) e Tellis (1986). Quando o varejista encontra formas de se diferenciar da concorrência, pode cobrar um preço maior pelos mesmos produtos e, consequentemente, aumenta suas vendas e margens de lucratividade. A

\section{Tabela 9 - Matriz de correlação entre objetivos de preço}

\begin{tabular}{|l|c|c|c|}
\hline & $\begin{array}{c}\text { Objetivos de } \\
\text { desenvolvimento de } \\
\text { mercado }\end{array}$ & $\begin{array}{c}\text { Objetivos de } \\
\text { realização de lucros e } \\
\text { vendas }\end{array}$ & $\begin{array}{c}\text { Objetivos de } \\
\text { competição }\end{array}$ \\
\hline Objetivos de desenvolvimento de mercado & 1,000 & 1,000 \\
\hline Objetivos de realização de lucros e vendas & 0,170 & 0,016 & 1,000 \\
\hline Objetivos de competição & 0,188 & 1 \\
\hline
\end{tabular}

\section{Tabela 10 - Preço por diferenciação e objetivos de preço}

\begin{tabular}{|l|c|c|c|c|}
\hline & Coeficiente $\beta$ (EP) & \multicolumn{3}{|c|}{ Intervalo de confiança de 95\% para Exp( $\beta$ ) } \\
\hline Objetivos de desenvolvimento de mercado & $0,025(0,102)$ & 0,839 & 1,025 \\
\hline Objetivos de realização de lucros e vendas & $0,119(0,104)$ & 0,918 & 1,126 \\
\hline Objetivos de competição & $0,620(0,094)$ & 1,546 & 1,859 \\
\hline Porcentagem total das predições corretas & $53,7 \%$ & & 2,234 \\
\hline Log Máxima verossimilhança (max) & 408,422 & & \\
\hline $\mathrm{R}^{2}$ de Nagelkerke & 0,033 & & \\
\hline$\chi^{2}$ melhoramento & 31,441, gl: 8 & & \\
\hline
\end{tabular}

Nota: $p<0,05$, EP - Erro Padrão; $g$ - graus de liberdade 
respectiva matriz de correlação entre os objetivos de preço é mostrada na Tabela 11, a seguir.

\section{Preço por localidade ou geografia}

Como pode ser visto na Tabela 12, a método de precificação por localidade ou geografia está associado com os objetivos de realização de lucro e vendas $(0,397)$ e objetivos de competição $(0,351)$.

De acordo com Seidler (1937), o método de precificação que considera a geografia ou a localidade onde o varejo é instalado procura satisfazer as necessidades de lucratividade da organização. Portanto, é perfeitamente compreensível sua associação com objetivos tanto de lucros e vendas quanto de competição. A respectiva matriz de correlação entre os objetivos de preço é mostrada na Tabela 13 , a seguir.

\section{Preço único}

A análise pertinente ao método de precificação por preço único (Tabela 14) revelou que os três objetivos de preços são associados a esse método: objetivos de realização de lucros e vendas $(0,698)$; objetivos de

\section{Tabela 11 - Matriz de correlação entre objetivos de preço}

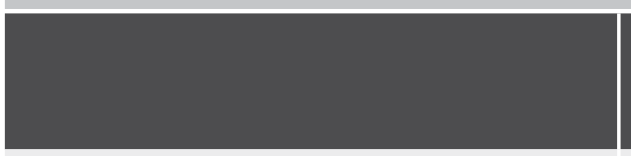

Objetivos de desenvolvimento de mercado

Objetivos de realização de lucros e vendas

Objetivos de competição
Objetivos de

desenvolvimento de

mercado

Objetivos de
realização de lucros
e vendas

1,000

0,196

0,174

Objetivos de

competição

\section{Tabela 12 - Preço por localidade ou geografia e objetivos de preço}

\begin{tabular}{|c|c|c|c|c|}
\hline & \multirow{2}{*}{ Coeficiente $\beta$ (EP) } & \multicolumn{3}{|c|}{ Intervalo de confiança de $95 \%$ para $\operatorname{Exp}(\beta)$} \\
\hline & & Inferior & $\operatorname{Exp}(\beta)$ & Superior \\
\hline Objetivos de desenvolvimento de mercado & $0,021(0,102)$ & 0,836 & 1,021 & 1,247 \\
\hline Objetivos de realização de lucros e vendas & $0,397(0,102)$ & 1,217 & 1,487 & 1,819 \\
\hline Objetivos de competição & $0,351(0,095)$ & 1,179 & 1,420 & 1,711 \\
\hline Porcentagem total das predições corretas & $54,7 \%$ & & & \\
\hline Log Máxima verossimilhança (max) & 406,911 & & & \\
\hline $\mathrm{R}^{2}$ de Nagelkerke & 0,039 & & & \\
\hline$\chi^{2}$ melhoramento & 38,226, gl: 8 & & & \\
\hline
\end{tabular}

Nota: $p<0,05, p<0,01$, EP - Erro Padrão; gl - graus de liberdade

Tabela 13 - Matriz de correlação entre objetivos de preço

\begin{tabular}{|l|c|c|c|}
\hline & $\begin{array}{c}\text { Objetivos de } \\
\text { desenvolvimento de } \\
\text { mercado }\end{array}$ & $\begin{array}{c}\text { Objetivos de } \\
\text { realização de lucros e } \\
\text { vendas }\end{array}$ & $\begin{array}{c}\text { Objetivos de } \\
\text { competição }\end{array}$ \\
\hline Objetivos de desenvolvimento de mercado & 1,000 & 1,000 \\
\hline Objetivos de realização de lucros e vendas & 0,135 & 0,011 & 1,000 \\
\hline Objetivos de competição & 0,159 & \\
\hline
\end{tabular}


desenvolvimento de mercado $(0,447)$; e objetivos de competição $(0,398)$. Esses resultados são condizentes com esse tipo de método, uma vez que, se o varejista define o preço, procura manter esse nível ao longo do tempo (HAWKINS, 1954).

A respectiva matriz de correlação entre os objetivos de preço é mostrada na Tabela 15, a seguir.

\section{Preço para entrar no mercado}

Quando se inicia a análise do método de preço para entrar no mercado, verifica-se que esse método de precificação está associado positivamente a dois objetivos de preço: objetivos de realização de lucros e vendas $(0,812)$; e objetivos de competição $(0,205)$. Esse é outro método de precificação cujos objetivos de preço condizem com o referencial teórico. Nesse método, a ideia é capturar mercado da concorrência e posterior realização de lucros (TELLIS, 1986).

A respectiva matriz de correlação entre os objetivos de preço é mostrada na Tabela 17, a seguir.

Baseada nos dados acima, a Tabela 18 descreve a associação entre os objetivos de preço e os métodos de precificação como identificada nesta pesquisa.

\section{CONCLUSÕES E RECOMENDAÇÕES}

Os objetivos deste trabalho de pesquisa foram investigar os objetivos de preço que as organizações de varejo perseguem, juntamente com os métodos de precificação que adotam, além de entender qual a medida de associação entre esses objetivos e os métodos de precificação. Coletando dados de 300 organizações de varejo, descobriu-se que as empresas da nossa amostra estão fundamentalmente perseguindo objetivos quantitativos no lugar de objetivos qualitativos, com uma forte ênfase na realização de vendas e lucros, seguidos pelo desenvolvimento de mercado para a organização.

Outro ponto importante na discussão, dentro dos métodos de varejo, é que as empresas da amostra parecem dar menor importância aos métodos baseados

\section{Tabela 14 - Preço único e objetivos de preço}

\begin{tabular}{|c|c|c|c|c|}
\hline & \multirow{2}{*}{ Coeficiente $\beta$ (EP) } & \multicolumn{3}{|c|}{ Intervalo de confiança de $95 \%$ para $\operatorname{Exp}(\beta)$} \\
\hline & & Inferior & $\operatorname{Exp}(\beta)$ & Superior \\
\hline Objetivos de desenvolvimento de mercado & $0,447(0,099)$ & 1,327 & 1,611 & 1,956 \\
\hline Objetivos de realização de lucros e vendas & $0,698(1,000)$ & 0,283 & 2,000 & 14,267 \\
\hline Objetivos de competição & $0,398(0,093)$ & 1,240 & 1,488 & 1,786 \\
\hline Porcentagem total das predições corretas & $50,3 \%$ & & & \\
\hline Log Máxima verossimilhança (max) & 415,888 & & & \\
\hline $\mathrm{R}^{2}$ de Nagelkerke & 0,007 & & & \\
\hline$\chi^{2}$ melhoramento & 32,884, gl: 8 & & & \\
\hline
\end{tabular}

Nota: $p<0,05$, EP - Erro Padrão; $g$ - graus de liberdade

Tabela 15 - Matriz de correlação entre objetivos de preço

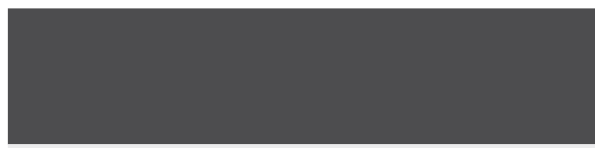

Objetivos de
desenvolvimento de
mercado

Objetivos de
realização de lucros e
vendas

Objetivos de competição

Objetivos de desenvolvimento de mercado

Objetivos de realização de lucros e vendas

Objetivos de competição

1,000

0,163

0,177

1,000

0,012

1,000 
nas expectativas do cliente. Essas empresas empregam com mais frequência o método tradicional, baseado nos custos do ofertante, provavelmente devido à simplicidade deste.

Também é interessante notar que a associação entre os objetivos de preços das organizações varejistas e os métodos de precificação adotados, identificados neste estudo, foi sempre positiva, em particular, no caso dos objetivos de realização de lucros e vendas, juntamente com os objetivos de competição.

Como implicações gerenciais, este estudo oferece uma melhor compreensão de como os objetivos de preço de uma organização varejista influenciam a adoção de um ou mais de um método de precificação. Sugere-se ainda, aos gestores das organizações varejistas, que tanto objetivos quantitativos quanto qualitativos sejam considerados na determinação dos objetivos de preço. Devido à concorrência acirrada no setor varejista e à grande maioria dos produtos ser considerada commodities, é importante, também, atentar-se aos métodos de precificação baseados nas expectativas do cliente. Marketing ou vendas podem contribuir fornecendo inputs baseados no mercado, enquanto outros departamentos, como o financeiro,

\section{Tabela 16 - Preço para entrar no mercado e objetivos de preço}

\begin{tabular}{|l|c|c|c|}
\hline & Coeficiente $\beta$ (EP) & \multicolumn{3}{|c|}{ Intervalo de confiança de 95\% para Exp( $\beta$ ) } \\
\hline Objetivos de desenvolvimento de mercado & Inferior & Exp( $\beta$ ) \\
\hline Objetivos de realização de lucros e vendas & $0,015(0,102)$ & 0,831 & 1,015 \\
\hline Objetivos de competição & $0,812(0,102)$ & 1,844 & 2,252 \\
\hline Porcentagem total das predições corretas & $0,205(0,094)$ & 1,020 & 1,227 \\
\hline Log Máxima verossimilhança (max) & $53,3 \%$ & & 1,478 \\
\hline$R^{2}$ de Nagelkerke & 408,72 & & \\
\hline$\chi^{2}$ melhoramento & 0,031 & & \\
\hline
\end{tabular}

Nota: $p<0,05$, EP - Erro Padrão; gl - graus de liberdade

\section{Tabela 17 - Matriz de correlação entre objetivos de preço}

\begin{tabular}{|l|c|c|c|}
\hline & $\begin{array}{c}\text { Objetivos de } \\
\text { desenvolvimento de } \\
\text { mercado }\end{array}$ & $\begin{array}{c}\text { Objetivos de } \\
\text { realização de lucros e } \\
\text { vendas }\end{array}$ & $\begin{array}{c}\text { Objetivos de } \\
\text { competição }\end{array}$ \\
\hline Objetivos de desenvolvimento de mercado & 1,000 & 1,000 \\
\hline Objetivos de realização de lucros e vendas & 0,153 & 0,014 & 1,000 \\
\hline Objetivos de competição & 0,203 & 1,000 \\
\hline
\end{tabular}

\section{Tabela 18 - Associação entre objetivos de preço e métodos de precificação}

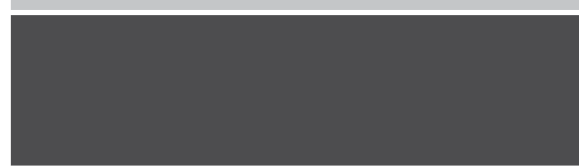

Objetivos de desenvolvimento de mercado

Objetivos de realização de lucros e vendas Objetivos de competição

\section{Preço para escoamento (venda rápida)}

Preço por diferenciação
Preço por localidade ou geografia
NS

$(+)$

$(+)$

NS

$+$

$+(+)$
$(+)$

(+)
NS

(+) NS

$+$

(t)

Preço único

Preço único

(+)

(+)

(+)
Preço para

entrar no

mercado
NS

(+)

(+) 
podem controlar as consequências dos custos e a lucratividade de cada decisão de preço. Portanto, uma implicação clara para os gestores responsáveis pela precificação em suas respectivas empresas é adotar uma abordagem sinergética quando desenvolvem suas estratégias de precificação, como uma atividade gerencial complexa que requer um exame cuidadoso do mercado no qual a empresa opera e dá as características únicas do seu ambiente interno.

Apesar de este trabalho de pesquisa representar uma tentativa de examinar empiricamente a relação entre objetivos de preço e métodos de precificação adotados pelo setor varejista, seus achados devem ser tratados com cautela. Mais especificamente, o contexto do estudo, a zona sul da cidade de São Paulo, é uma óbvia limitação do trabalho, já que essa realidade de mercado não pode ser generalizada para outras cidades ou regiões do País.

Como sugestão para futuros estudos, pesquisadores podem investigar o impacto que o contexto da organização varejista, como tamanho ou setor de operação, possui nas variáveis ambientais. Ou, ainda, investigar se organizações varejistas que atendem diferentes classes sociais possuem objetivos e métodos de precificação diferenciados e quais outras associações podem ser descobertas. Outra possibilidade seria replicar este estudo em contextos regionais diferentes. Um estudo considerando empresas que aleguem ter posicionamento de marketing definido e alguma estratégia de diferenciação também seria interessante para compreender o processo de estabelecer estratégias de preço orientadas para o cliente. O exame detalhado dessas variáveis pode levar a um melhor entendimento dos conceitos apresentados neste estudo.

\section{REFERÊNCIAS}

ANDERSON, E. T; SIMESTER, D. I. Effects of $\$ 9$ price endings on retail sales: evidence from field experiments quantitative. Marketing and Economics, v. 1, n. 1, p. 93-110, 2003.

BEDÊ, M. A. Onde estão as micro e pequenas empresas no Brasil. São Paulo: Serviço Brasileiro de Apoio às Micro e Pequenas Empresas, 2006.

BERRY, L. L; YADAV, M. S. Capture and communicate value in the pricing of services. MIT Sloan Management Review, v. 37, n. 4, p. 41-51, 1996.
BONNICI, L. H. Pricing dimensions in health-care service. Journal of Professional Services Marketing, v. 8, n. 1, p. 92-98, 1991.

BRAY, J; HARRIS, C. The effect of nine-ending prices on retail sales: a quantitative UK based field study. Journal of Marketing Management, v. 22, n. 5, p. 601-616, 2006.

Calantone, R. J; Di BEnEDetTo, A. C. Clustering product launches by price and launch strategy. Journal of Business E Industrial Marketing, v. 22, n. 1, p. 4-19, 2007.

CHEN, Y; MOORTHY, S; ZHANG, Z. J. Research note price discrimination after the purchase: rebates as statedependent discounts. Management Science, v. 51, n. 7, p. 1131-1140, 2005.

CRAVENS, K. S; GLOVER, H. D. Pricing complexities in the pharmaceutical industry: implications for external auditors. Managerial Auditing Journal, v. 10, n. 7, p. 8-16, 1995.

DeGRABA, P. The loss leader is a turkey: targeted discounts from multi-product competitors. International journal of industrial organization, v. 24, n. 3, p. 613-628, 2006.

DIAMANTOPOULOS, D. Pricing: theory and evidence - a literature review. In: BAKER, M. J. (Ed). Perspectives on marketing management. Chichester: John Wiley \& Sons, 1991.

ENTENBERG, R. Suggested changes in census classifications of retail trade. Journal of Marketing, v. 24, n. 3, p. 39-43, 1960.

EVANS, K. R; BELTRAMINI, R. F. A theoretical model of consumer negotiated pricing: an orientation perspective. Journal of Marketing. v. 51, n. 2, p. 58-73, 1987.

FIELD, A. Descobrindo a estatística usando o SPSS. Porto Alegre: Artmed, 2009.

GUILTINAN, J. P. The price bundling of services: a normative framework. Journal of Marketing, v. 51, n. 2, p. 74-85, 1987.

GUTHRIE, J. A. Impact of geographical price discrimination on the buyer. Journal of Marketing, v. 14, n. 4, p. 538$543,1950$.

HAIR, J. F. Jr. e outros. Análise multivariada de dados. Porto Alegre: Bookman, 2009. 
HANSEN, T; SOLGAARD, H. S. Strategic pricing: fundamental considerations and future perspectives. The Marketing Review, v. 4, p. 99-111, 2004.

HAWKINS, E. R. Price policies and theory. Journal of Marketing. v. 18, n. 3, p. 233-240, 1954.

HERZOG, R. A; BACHMAN, J. G. Effects of questionnaire length on response quality. Public Opinion Quarterly, v. 45, n. 4, p. 549-559, 1981.

HESS, J. D; GERSTNER, E. Loss leader pricing and rain check policy. Marketing Science, v. 6, n. 4, p. 358-374, 1987.

HOFFMAN, K. D; BATESON, J. E. G. Principios de marketing de serviços: conceitos, estratégias e casos. São Paulo: Cengage, 2010.

HORNBY, W. B; MaCLEOD, M. Pricing behaviour in the Scottish computer industry. Management Decision, v. 34, n. 6, p. 31-42, 1996.

HOWARD, C; HERBIG, P. Japanese pricing policies. The Journal of Consumer Marketing, v. 13, n. 4, p. 5-17, 1996.

INDOUNAS, K; AVLONITIS, G. J. Pricing objectives and their antecedents in the services sector. Journal of Service Management, v. 20, n. 3, p. 342-374, 2009.

JAIN, S. Marketing planning and strategy. Mason: Thomson Custom, 2004.

KEHAGIAS, J; SKOURTIS, E; VASSILIKOPOUlOU, A. Plaiting pricing into product categories and corporate objectives. Journal of Product \& Brand Management, v. 18, n. 1, p. 67-76, 2009.

KEIL, S. K; REIBSTEIN, D; WITTINK, D. R. The impact of business objectives and the time horizon of performance evaluation on pricing behavior. International Journal of Research in Marketing, v. 18, n. 1, p. 67-81, 2001.

KRZANOWSKI, W. J. Statistical principles and techniques in scientific and social investigations. New York: Oxford University, 2007.

KUKAR-KINNEY, M; RIDGWAY, N. M; MONROE, K. B. The role of price in the behavior and purchase decisions of compulsive buyers, Journal of Retailing, v. 88, n. 1, p. 63-71, 2012
LAL, R. Consumer expectations and loss-leader pricing in retail stores. Working Papers. Stanford Graduate School of Business, p. 1-48, 1991.

LAL, R; MATUTES, C. Retail pricing and advertising strategies. Journal of Business. v. 67, n. 3, p. 345-370, 1994.

LAN, X; KUKAR-KINNEY, M; MONROE, K. B. 'Effects of consumers' efforts on price and promotion fairness perceptions. Journal of Retailing, v. 86, n. 1, p. 1-10, 2010.

LANGEARD, E. Specificity of the Pricing Policy in Service Activities, In: 6th International Research Seminar in Service Management, 6., 2000, Le Londe Les Maures, Proceedings..., Le Londe Les Maures, France: IAE, 2000.

LANZILLOTTI, R. F. Pricing objectives in large companies. American Economic Review, v. 48, n. 5, p. 921-40, 1958.

LEVY, M. e outros. Emerging trends in retail pricing practice: implications for research. Journal of Retailing, v. 80, n. 3, p. 13-21, 2004.

MACÉ, S. The impact and determinants of nine-ending pricing in grocery retailing original research article. Journal of Retailing, v. 88, n. 1, p. 115-130, 2012.

MALHOTRA, N. K. Pesquisa de marketing: uma orientação aplicada. Porto Alegre: Bookman, 2001.

MANNING, K. C; SPROTT, D. E. Multiple unit price promotions and their effects on quantity purchase intentions. Journal of Retailing, v. 83, n. 4, p. 411-412, 2007.

MARINESCU, P; MIHAI, N. S; SORIN, T. Pricing strategy used as a tool for building customer satisfaction in the retail sector. Annals of Faculty of Economics, University of Oradea, Faculty of Economics, v. 1, n. 2, p. 1122-1127, 2010.

MARN, M. V; ROSIELLO, R. L. Managing price, gaining profit. Harvard Business Review, v. 70, n. 5, p. 84-94, 1992.

MATZLER, K; HINTERHUBER, H. H. How to make product development projects more successfully by integrating Kano's model of customer satisfaction into quality function deployment. Tecnovation, v. 18, n. 1, p. 25-38, 1998.

MOORE, S. T. Pricing: a normative strategy in delivery of human services. Social Work, v. 40, n. 4, p. 473-481, 1995. 
MORRIS, M. H; CALANTONE, R. J. Redefining the purchasing function: an entrepreneurial perspective. International Journal of Purchasing and Materials Management, v. 27, n. 4, p. 2-9, 1991.

MUNGER, J. L; GREWAL, D. The effects of alternative price promotional methods on consumers' product evaluations and purchase intentions. Journal of Product and Brand Management, v. 10, n. 3, p. 185-197, 2001.

NOBLE, P. M; GRUCCA, T. S. Industrial pricing and managerial practice. Marketing Science, v. 18, n. 3, p. 435-454, 1999.

NUNNALLY, J. C. Psychometric theory. 2. ed. New York: McGraw, 1978.

OXENFELDT, A. R. Pricing decisions: how they are made and how they are influenced. Management Review, v. 72, n. 11, p. $23-25,1983$.

POPESCU, I; WU, Y. Dynamic pricing strategies with reference effects. Operations Research, v. 55, n. 3, p. 413429, 2007.

RAO, A. R; MONROE, K. B. Causes and consequences of price premiums, Journal of Business, v. 69, n. 4, p. 511535. 1996.

RATZA, C. L. A client-driven model for service pricing. Journal of Professional Services Marketing, v. 8, n. 2, p. 55-64, 1993

SEIDLER, G. Geographical price relations and competition. Journal of Marketing, v. 1, n. 3, p. 198-204, 1937.

SHAH, N. H; DIXIT, V. M. Price discount strategies: a review. Revista Investigación Operacional, v. 26, n. 1, p. 19-32, 2005.

SHIPLEY, D. D. Pricing objectives in British manufacturing industry, Journal of Industrial Economics, v. 29, n. 4, p. 429-443, 1981.

SHIPLEY, D. D; JOBBER, D. Integrative pricing via the pricing wheel. Industrial Marketing Management, v. 3, n. 3, p. 301-314, 2001.

SHY, O. How to price: a guide to pricing techniques and yield management. Cambridge: Cambridge University, 2008.
SIMON, H. Pricing opportunities and how to exploit them. Sloan Management Review, v. 33, n. 2, p. 52-62, 1992.

SIRGY, M. J; DONG-JIN, L. Setting socially responsible marketing objectives: a quality-of-life approach. European Journal of Marketing, v. 30, n. 5, p. 20-34, 1996.

SMITH, G. E. Managerial pricing orientation: the process of making pricing decisions. Pricing Strategy and Tactics, v. 3, n. 3, p. 28-39, 1995.

TAHER, A; BASHA, H. Heterogeneity of consumer demand: opportunities for pricing of services. Journal of Product \& Brand Management, v. 15, n. 4/5, p. 331340, 2006.

TELLIS, G. J. Beyond the many faces of price: an integration of pricing strategies. Journal of Marketing, v. 50, n. 4, p. 146-160, 1986.

THEOTOKIS, A; PRAMATARI, K; TSIROS, M. Effects of expiration date-based pricing on brand image perceptions original research article. Journal of Retailing, v. 88, n. 1, p. 72-87, 2012.

THOMAS, M; MORWITZ, V. Penny wise and pound foolish: the left-digit effect in price cognition. Journal of Consumer Research, v. 32, n. 1, p. 54-64, 2005.

TOledO G. L; PROENÇA, M. C. A; MELlO Jr, S. B. Política de preços e diferencial competitivo: um estudo de casos múltiplos na indústria de varejo. RAUSP-Revista de Administração da Universidade de São Paulo, v. 41, n. 3, p. 324-338, 2006.

VARKI, S; COLGATE, M. The role of price perceptions in an integrated model of behavioral intentions. Journal of Service Research, v. 3, n. 3, p. 232-240, 2001.

YEOMAN, I; MCMAHON-BEATTIE, U. Luxury markets and premium pricing. Journal of Revenue and Pricing Management, v. 4, n. 4, p. 319-328, 2006.

ZIELKE, S. Measurement of retailers' price images with a multiple-item scale. The International Review of Retail, Distribution and Consumer Research, v. 16, n. 3, p. 297316, 2006.

ZIELKE, S. How price image dimensions influence shopping intentions for different store format, European Journal of Marketing, v. 44, n. 6, p.748-770, 2010. 